\title{
Sken\&agraphie
}

SKISingraphil Coulisses des arts du spectacle et des scènes

émergentes

5 | 2018

Juste la fin du monde, de Lagarce à Dolan

\section{Autour des films vidéo}

Entretien avec François Berreur, Catherine Derosier-Pouchous et Patrick Zanoli

\section{Pascal Lécroart et Alexis Leprince}

\section{OpenEdition}

\section{Journals}

Édition électronique

URL : http://journals.openedition.org/skenegraphie/1429

DOI : 10.4000/skenegraphie.1429

ISSN : 2553-1875

\section{Éditeur}

Presses universitaires de Franche-Comté

\section{Édition imprimée}

Date de publication : 1 janvier 2018

Pagination : 111-124

ISBN : 978-2-84867-5609-8

ISSN : $1150-594 X$

Référence électronique

Pascal Lécroart et Alexis Leprince, «Autour des films vidéo », Sken\&agraphie [En ligne], 5 | 2018, mis en ligne le 01 janvier 2019, consulté le 03 mai 2019. URL : http://journals.openedition.org/ skenegraphie/1429; DOI : 10.4000/skenegraphie.1429

Ce document a été généré automatiquement le 3 mai 2019.

Presses universitaires de Franche-Comté 


\title{
Autour des films vidéo
}

\author{
Entretien avec François Berreur, Catherine Derosier-Pouchous et Patrick \\ Zanoli
}

Pascal Lécroart et Alexis Leprince

À l'occasion de la journée d'études «Juste la fin du monde: des premiers brouillons à l'adaptation cinématographique» dont le carnet critique se fait ici l'écho, furent également diffusés les deux films vidéo de Jean-Luc Lagarce, Journal 1 et Portrait, réalisés avec l'aide $\mathrm{du} \mathrm{CICV}^{1}$ et son directeur Pierre Bongiovanni. Trois témoins de la réalisation de ces films nous parlent de cette expérience cinématographique menée par Jean-Luc Lagarce: François Berreur, metteur en scène, directeur littéraire des éditions Les Solitaires intempestifs, acteur et assistant de Jean-Luc Lagarce, et qui apparait à de nombreuses reprises dans Journal 1; Catherine Derosier-Pouchous, responsable des productions au musée du Louvre, qui s'est occupée de la production des films au CICV et les a notamment accompagnés dans de nombreux festivals; enfin Patrick Zanoli, monteur, qui a effectué avec le dramaturge un travail de longue haleine au CICV.

Vous SOUVENEZ VOUS DE LA PREMIÈre fOIS OÙ JEAN-LUC LAgARCE VOUS A PARLÉ dU PROJET DU fILM JOURNAL 1 ? COMMENT L'A-T-IL ÉVOQUÉ AVEC VOUS ?

Catherine Derosier-Pouchous : La première fois qu'il a évoqué ce projet, ce n'était pas avec nous, c'était avec Pierre Bongiovanni, le directeur du CICV. Pierre m'en a très vite parlé, et ce que je savais, c'est que Jean-Luc Lagarce avait une carte blanche pour partir avec une caméra et faire le film qu'il souhaitait faire sans avoir besoin de justifier son projet, même s'il avait des idées. Le CICV était aussi un lieu pour ça, un lieu d'expérimentation et de création. On avait par ailleurs une relation depuis très longtemps avec Jean-Luc Lagarce: Pierre Bongiovanni, Patrick Zanoli et moi-même, nous avons travaillé ensemble dans un centre d'action culturelle à Montbéliard, et, quand Jean-Luc Lagarce a créé sa compagnie, la Roulotte, nous avons collaboré à ses projets. Nous avions déjà cette relation depuis très longtemps autour du théâtre, tout s'est donc fait naturellement. Ce qui est sûr, c'est qu'il avait une vraie carte blanche. 
Ce type d'expérience étalt-IL pour le centre un CAS singulier ou UNe pratique HABITUELLE ?

Catherine Derosier-Pouchous : Rappelons que c'était un centre de création, avec une résidence d'artistes, des moyens apportés par l'État et par la région, afin qu'il puisse expérimenter des formes nouvelles et novatrices autour des questions d'images animées, de productions, d'installation et de télévision. Ce n'était donc pas un cas à part; c'était habituel de pouvoir permettre à des créateurs d'avoir un espace de production et un outil au CICV.

Patrick Zanoli : L'objectif était aussi de laisser du temps aux artistes, le temps qu'il fallait à la réalisation de leur projet. Il n'y avait pas vraiment de limites, et Jean-Luc Lagarce est parti un jour avec une caméra et une trentaine de cassettes vidéo qu'on lui avait fournies; il est parti plusieurs mois, et il est revenu me voir avec toutes ses cassettes enregistrées. Il y avait plus de trente heures ou quarante heures de rushes. On en a visionné quelques-unes avec Pierre, et on a vu qu'il y avait quelque chose d'important à réaliser autour du travail qu'avait fait Jean-Luc Lagarce en tournage. Le plus frappant, c'était qu'il n'avait jamais filmé auparavant, mais qu'il montrait déjà un grand professionnalisme en termes de cadrage notamment, où il n'y avait rien d'amateur. Il insistait, il restait longtemps sur les personnages, même si les personnages sortaient, il les laissait revenir dans le cadre, cela nous avait intéressé. C'était cette liberté-là qu'avaient les artistes au CICV, et beaucoup de temps.

l'Aspect le plus frappant du film, C'est Ce montage dont on deVine Qu'il a NÉCESSITÉ BEAUCOUP de traVAIL. ANNE UBERSFELd CARACTÉRISE LE THÉÂTRE PAR SA POlyphonie informationnelle, et IL SEMble QUE, SUR Le plan technique, dans JOURNAL 1, ON EST ÉgALEMENT FACE À UNE VRAIE POLYPHONIE, EXPLORANT BEAUCOUP DE POSSIBILITÉs VISUELLES ET SONORES, SANS PARLER DES TEXTES SUPERPOSÉS AUX CAPTATIONS. LORSQU'ON REgARde POUR LA PREMIÈre FOIS LE film, ON EST QUELQUE PEU PERdU CAR ON Ne SAIT PAS S'IL FAUT NE LIRE QUE LE TEXTE OU AU CONTRAIRE SE LAISSER PRENDRE PAR LES IMAgES, OU ENCORE SI C'EST FAIT POUR QUE L'ON PASSE LIBREMENT DE L'UN À L'AUTRE EN CONSTITUANT SA PROPRE EXPLORATION. DES ÉLÉMENTS DE LA BANDESON SONT RÉCURRENTS, D'AUTRES NON ; LA MUSIQUE EST PARFOIS PRISE EN SON DIRECT,

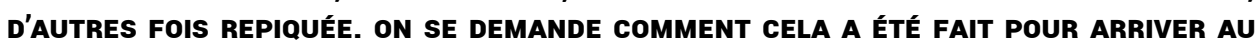
RÉsultat final À PARTiR de CET ENSEMble de CASSEtTes.

Patrick Zanoli : Jean-Luc Lagarce a dérushé toutes les images et a choisi toutes les images et les sons qui devaient intervenir dans le film. Le CICV était équipé de matériel dernier cri pour l'époque; c'était une des structures institutionnelles les mieux équipées en France - on n'avait pas l'ordinateur à l'époque, c'est venu un peu après. Pour travailler ce type d'images, on disposait d'énormes magnétoscopes numériques mais qui permettaient justement de mélanger plusieurs informations d'images, de sons, et de créer des collages vidéo. Toute l'architecture du film a été décidée par Jean-Luc Lagarce, avec tous ces sons qui devaient revenir ou être mis en avant. C'est lui qui avait décidé de cette structure complexe. Ce n'est pas un film simple à regarder, si on rate des choses, on les a ratées, c'est un peu comme...

Catherine Derosier-Pouchous : ...dans la vie !

Patrick Zanoli : Comme dans la vie, exactement. On peut privilégier de lire le texte, ou de perdre le texte et de se concentrer sur les images, c'est un choix du spectateur. Je crois que Jean-Luc Lagarce ne voulait imposer aucune règle pour voir son film. 
Catherine Derosier-Pouchous : Jean-Luc Lagarce m'a dit que cela avait été un travail de dentellier, que Patrick a fait avec lui mais que Patrick a fait aussi seul : avec le travail théâtral, Jean-Luc Lagarce partait, revenait ; Patrick travaillait des séquences et les lui montrait, et Jean-Luc validait ou non. Il savait ce qu'il souhaitait au niveau du rendu, de la narration, du rythme, de l'ambiance du film, mais il fallait quand même trouver des solutions visuelles, techniques, et d'articulations, donc il fallait avoir une grande maîtrise technique des outils. Il y a eu beaucoup d'allers-retours et le montage a duré très longtemps; je me souviens de longues journées et de longues nuits. Ces allersretours étalés dans le temps permettaient de mûrir les séquences, de les réfléchir, de les digérer. Il y a eu aussi un vrai dialogue avec Patrick. Il m'avait dit que Patrick était beaucoup plus généreux que lui sur les gens. Il avait un peu la dent dure parfois!

Jean-luc lagarce s'est promené aVec une caméra pendant plusieurs mois, près de deuX ans au total. Comment cette présence de la caméra a-t-elle été vécue PAR SES PROCHES? Y AVAIT-IL UNE SORTE dE gÊNE, DE DISTANCE, D'AMUSEMENT ?

François Berreur : Ce qu'il faut dire, c'est que la caméra était comme ça (il montre une très grande taille), ce n'était pas un petit caméscope! C'était un objet qui pour lui induisait un rapport presque sportif, et peu discret, sauf peut-être de loin, mais en effet quand il était dans une pièce, ce n'était pas du tout le même rapport ; l'objet lui-même empêchait l'oubli ou la discrétion. C'était surprenant ou encombrant, on était plus ou moins disponibles mais je crois qu'on s'est prêté au jeu.

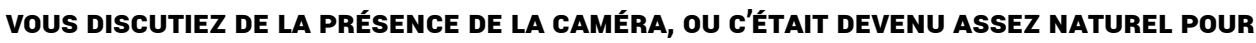 vous?}

François Berreur : Non, ce n'était pas naturel, mais cela faisait partie d'un contexte plus global : à ce moment-là, Mireille et moi étions les deux personnes qu'il voyait le plus souvent car nous étions en tournée sur le spectacle Les Égarements du cœur et de l'esprit. Ensuite, il a été beaucoup seul lorsqu'il a amené la caméra à Berlin. Quand il nous filme, ce n'est pas à proprement parler naturel mais cela prend place dans un rapport au travail où l'on se voyait souvent : pour la tournée des Égarements du cœur et de l'esprit, nous étions trois ; on arrivait parfois à cinq heures du matin sans technicien, on déchargeait le camion, on jouait, on chargeait à nouveau ; c'était professionnel mais c'était un autre rapport au travail qu'après son retour de Berlin. Ce film est comme le moment charnière d'une époque : quand il commence à filmer, ce sont les tournées qu'on fait plutôt en région, et il continue de filmer pendant son séjour à Berlin où il écrit Juste la fin du monde, qui est le moment de l'échec sublime et désespérant de l'écrivain Jean-Luc Lagarce. Après cette période, il montera La Cantatrice chauve et rencontrera le succès en tant que metteur en scène. Ce film-là est témoin d'un basculement entre un professionnalisme fragile et un professionnalisme solide.

Lorsque le montage a été fini et que les premières personnes ont vu le film, certaines personnes, des proches, ont découvert qu'il était séropositif. C'est pour cela que je lui disais que parfois il y allait un peu fort - ce qu'il note dans son Journal. C'était à ce propos qu'on pouvait discuter par rapport au film. Le film et le montage amènent une dimension très fictionnelle, mais à partir d'une matière qui est celle de l'image très intime et réelle. C'est pourquoi parfois les gens ne voyaient pas le film, ils voyaient que Jean-Luc Lagarce était séropositif. Je me souviens qu'à Montbéliard, où un public plus large assistait à la première projection, ça tanguait un peu !

CE QUI EST FRAPPANT ÉgALEMENT DANS LE film, C'EST L'ANCRAgE HISTORIQUE SOUVENT EXPLICITEMENT AFfiRMÉ, AVEC L'ÉVOCATION DES ÉLECTIONS EN FRANCE ET LA MONTÉE DU 
FRONT NATIONAL, LE MUR DE BERLIN, LA TOMBE DE BRECHT AVEC L'INSCRIPTION « JUDE RAUS", ETC. CETTE VOLONTÉ de PORTER UN tÉmoIgNage historique ÉtAit-elle REVENDIQUÉE PAR JEAN-LUC LAgARCE ?

François Berreur: Oui, c'est un film dans lequel, il retrouve, à travers son regard cinématographique, toutes les thématiques qu'il brasse dans son œuvre. Par exemple, lorsqu'à Berlin il filme les tombes taguées du cimetière juif, il rejoint les préoccupations de l'un de ses premiers textes, Vagues Souvenirs de l'année de la peste, qui porte ce souci de la montée de l'extrémisme.

En plus, il est à Berlin dans un moment historique. Entre le moment où il a obtenu la bourse et le moment où il arrive à Berlin, le mur est tombé! En même temps, Berlin, cela rejoint aussi son goût pour la littérature allemande, toute la mythologie Mitteleuropa dont on peut dire que la première partie de son œuvre est très nourrie. Il y a donc une conjonction thématique et historique, mais, dans le même temps, je crois qu'il est clair qu'il n'a pas la caméra pour filmer uniquement son quotidien. Il a une conscience beaucoup plus large : ce qu'il filme, c'est un document, mais ce qu'il veut raconter, c'est une chose beaucoup plus universelle, donc ce n'est pas du hasard. Il utilise le hasard des événements pour les mettre dans son point de vue universel.

Patrick Zanoli : L'actualité l'intéressait énormément, tout ce qui se passait à cette période-là l'intéressait, il en parlait souvent. Autant il ne parlait pas de SIDA, autant il parlait de politique, il dévorait Libération tous les jours sans rater un jour, et ensuite il nous parlait de ce qu'il avait lu pendant qu'on montait avec beaucoup d'humour d'ailleurs, en général. Il n'était pas déconnecté de la réalité et vivait cela très fortement.

François Berreur : Mais je pense que, grosso modo, s'il avait filmé sur une autre période historique, le film aurait raconté à peu près la même chose : on voit les tournées, cela aurait pu être d'autres tournées; l'écriture, il a écrit à Berlin, ce sont des circonstances particulières, mais il aurait sûrement trouvé autre chose à Paris, ailleurs. Il n'aurait peut-être pas raconté de la même manière, ni avec les mêmes images, mais je pense que ce qu'il avait envie de raconter à ce moment-là, ce n'était pas lié à cette période historique spécifiquement. Il n'a pas fait le film avec ce qu'il a vu, mais avec ce qu'il avait dans la tête.

Catherine Derosier-Pouchous : Lors des entretiens que j'ai menés avec lui après le film, il me disait qu'il était très surpris de la réaction des gens, qui était disproportionné selon lui. Le fait de découvrir qu'il avait le SIDA obstruait toute la vision que le public pouvait avoir du film, alors qu'en fin de compte, ce qui comptait pour lui, c'était la forme, le travail de création. Bien évidemment il joue avec la révélation personnelle, l'expression de son histoire et de sa souffrance.

Je me souviens très bien de discussions où à la fois il disait qu'il ne s'exposait pas dans le film, il exposait plus les autres - ce qui n'était pas vrai pour moi. Pour lui, c'était la forme qui comptait et c'était ce récit de sa vie, comme elle est, qu'il voulait partager mais que ce n'était pas vraiment un sujet en soi. Après tout, à d'autres époques, on mourrait d'autres maladies; ça faisait partie de la vie. Il avait beaucoup de distance pour rester élégant.

En même temps, je pense que c'est très manipulatoire : c'est de la narration et c'est une mise en scène ; manipulatoire au sens positif, c'est-à-dire qu'au cinéma, on manipule des images et des sons. Le texte de Journal 1 donne quand même du sens aux images, parce qu'on aurait pu montrer les mêmes images et nous raconter autre chose. Donc je crois qu'il est clair qu'il a fait le choix d'associer ce moment du Journal où il apprend 
qu'il est séropositif, ce moment qui change complètement sa perception de la vie et son regard sur ce qui est important, ce qu'a été sa vie et ce que cela va être, c'est en filigrane. Et en même temps, il joue avec ça, ce n'est pas tout à fait de cela dont il est question.

François Berreur: Oui, parce que les textes du Journal sont datés de 1988; or le tournage ne commence qu'en 1990. Si on voit le film et qu'on ignore cela, on peut avoir l'impression que le texte colle aux images chronologiquement; on croit qu'il était en tournée et qu'il apprend ce jour-là qu'il est séropositif, à cet endroit-là... mais pas du tout, c'était deux ans avant ! Il amène sur un présent deux choses qui n'ont rien à voir.

Le récit est fictionné à partir de choses exactes, d'autant plus qu'il est construit à partir d'un journal. Mais ce journal est à l'envers : c'est-à-dire que lui, Jean-Luc Lagarce, est rarement filmé, mis à part quand il se filme dans la glace; ceux qu'on filme, ce sont toujours les autres. Les images montrent toujours ce qui fait l'extérieur de sa vie mais jamais sa vie.

Le paradoxe du texte, c'est qu'il renvoie à sa vie, sauf que c'est écrit et que les événements qu'il raconte ont eu lieu deux ans auparavant. Néanmoins, il y a un moment donné dans le film où les événements du texte et ceux de l'image concordent : le film débute avec deux ans d'écart entre le texte et l'image, et à la fin texte et image sont raccords. Le film est construit sur cette distorsion temporelle.

Quand l'on voit les images de Berlin, avec sa machine à écrire, ce qu'il filme, c'est l'écriture de Juste la fin du monde. Le texte débute en 1988, lorsqu'il fait les premiers synopsis de Juste la fin du monde, et la fin du film, c'est la fin de l'écriture de la pièce. On peut dire qu'il y a le film de ce qu'il a vécu pendant l'écriture de la pièce.

Catherine Derosier-Pouchous : C'était ce qu'il souhaitait, en effet.

POURTANT, ON A L'IMPRESSION QUE JUSte LA fin du MONDE FONCTIONNE PLUTÔt COMME UNE ÉPURE DE THÉÂTRE, AVEC UNE gRANDE SIMPLICITÉ ET UNE ORgANISATION TRÈS STRICTE, UNE FORME D'ÉCONOMIE DE MOYENS. OR JOURNAL 1 SEMBLE PROPOSER UN TOUT AUTRE ASPECT DE SON CARACTÈRE, AVEC CE RAPPORT AU MONTAgE, À LA SUPERPOSITION, À L'eXcès d'informations. est-ce que cela dit quelque chose de lagarce comme CrÉateur? le pays LOINTAIN FONCTIONNERA Également PAR AJOUts ET SUPERPOSITIONS PAR EXEMPLE.

François Berreur : Je dirais que c'est le projet cinématographique, et l'endroit même du CICV qui lui a permis d'exprimer ce rapport-là entre le texte et l'image, grâce au travail de montage incroyable pour l'époque. La qualité d'image n'apparait pas bonne aujourd'hui, c'est dommage car le rapport au montage, avec les outils de l'époque, reste encore aujourd'hui d'une grande modernité.

LE SECONd film Qu'Il A FAIT, PORTRAIT, EST LUI TRÈs diffÉRENT, TRÈs COURT, ET CETTE FOIS-CI JEAN-LUC LAgARCE APPARAÎT NETTEMENT À L'ÉCRAN, ALORS QUE DANS JOURNAL 1 SA PRÉsence ÉtAit très discrète. QUELLe diffÉrence y A-T-IL ENTRE SES deUX DÉMARCHES? A-T-IL VOULU FAIRE DEUX fiLMS DIFFÉRENTS OU DEUX fILMS QUI SE COMPLÈTENT ?

Catherine Derosier-Pouchous : Le contexte est le suivant: le film Journal 1 a été présenté dans un festival au Brésil, où je l'ai accompagné. Or, parmi les organisateurs du festival, il y avait quelqu'un qui avait créé au Brésil un festival de films d'une minute, dont le CICV était partenaire. Quand je suis rentré du Brésil, j'ai raconté à JeanLuc la projection du film : les gens là-bas étaient persuadés que c'était une fiction. Un débat avait eu lieu puisque pour eux c'était mis en scène, ça ne pouvait pas être un 
témoignage de la réalité, on n'était ni dans le documentaire, ni dans l'essai. Jean-Luc pensait que cet effet était dû à la distance, et je lui avais proposé de retourner au Brésil en réfléchissant à l'exercice technique de raconter quelque chose en une minute, ce qui est très dur. J'ignore comment il a lié les choses dans son esprit entre les deux films mais en tout cas il avait envie de continuer à faire des films. Le film est allé en compétition et il a gagné le premier prix. Il en était extrêmement fier !

Patrick Zanoli : Portrait, ce sont des images fixes de Jean-Luc Lagarce, de sa petite enfance jusqu'à une période plus contemporaine du moment du film, et quelques textes qui apparaissent. Je n'ai pas fait le montage mais on en avait parlé pour voir comment on pouvait passer des premières images aux dernières.

Catherine Derosier-Pouchous : C'est un montage de photos en fait, des images fixes qui devaient être animées par un discours.

Patrick Zanoli : Jean-Luc Lagarce était très curieux, la découverte qu'il a fait de ces nouveaux outils numériques l'intéressait beaucoup, et c'était cette curiosité-là qui a donné lieu à Journal 1 : on aurait pu très bien partir sur quelque chose de beaucoup plus classique en termes de formes de montage, avec une ou plusieurs fois off par exemple. Je crois que dans le film il y a un ou deux cuts seulement, le reste c'est du passage d'images. Ce film était un peu un ovni à l'époque. La difficulté était de tenir le rythme, et nous avions beaucoup de débats entre nous pour trouver les bonnes vitesses de texte et l'arrivée de certaines images qui faisaient de nouveau partir le rythme, l'intensité, la dramaturgie du film. Il était très curieux de découvrir comment on pouvait écrire avec ces nouveaux outils ; c'était plus un travail d'écriture qu'un travail cinématographique.

François Berreur : C'est vraiment la commande qui a fait le film d'une minute. Après, cela fait partie aussi de la préoccupation de la fin, de ce que l'on raconte de sa vie, de sa biographie, ce qu'on laisse : les archives, où tout est bien rangé, témoignent de cela. Néanmoins, à la fin de sa vie, il était en forme, pas dans l'abandon, mais en train de réfléchir, de créer; il répétait encore, et il est mort quelques jours après avoir fini $L e$ Pays lointain. Cette préoccupation de la fin n'était pas forcément une chose qui le minait, mais au contraire cela avait plutôt tendance à le dynamiser.

Ce film fait donc partie d'une réflexion plus globale. Ces deux films s'inscrivent dans le contexte de l'échec de Juste la fin du monde et du succès des mises en scène de Jean-Luc Lagarce. Une autre voie s'ouvre, et l'écriture, c'est le désert pendant des années. Même quand il fait Les Règles du savoir-vivre, est-ce vraiment un texte de lui ? Juste la fin du monde était pour lui un texte de maturité. Il n'écrivait que depuis dix ans, et cette pièce allait au bout de quelque chose, d'une inclination, qu'il reprendra avec J'étais dans ma maison et j'attendais que la pluie vienne et Le Pays lointain. À ce moment-là, le travail cinématographique avait une part toute aussi importante que la mise en scène pour lui, contrairement à l'écriture qu'il avait laissé de côté après Juste la fin du monde. L'achèvement des films et la reconnaissance qu'il en a eue étaient donc très importants pour lui.

Catherine Derosier-Pouchous : D'ailleurs, il avait un nouveau projet de film, et le CICV allait le produire: il avait trouvé deux albums de photographies, aux puces, qui montraient une photo répétée à l'infini du même personnage, une femme, dans toutes ses situations de vie, dans les années 1950 ou 1960. Il avait le projet à partir de là de construire une nouvelle narration et un nouveau film. Il était dans une dynamique et 
ces outils d'écriture audiovisuelle lui permettaient d'être créatif et reconnu dans une écriture.

François Berreur : De plus, il écrivait un scénario en parallèle. L'idée du cinéma n'était pas absente en lui, et il commençait à former une sorte de totalité artistique.

Concernant le travall textuel, Jean-luc lagarce a Repris les textes de son JOURNAL SUR UNE PÉRIODE DONNÉE, QU'IL A RÉÉCRIT...

François Berreur : Non, pas du tout, il a fait du copier-coller depuis le Journal, il ne s'est pas fatigué, il a coupé un peu, c'est tout !

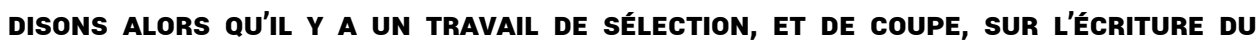

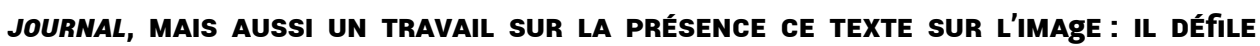
PARFOIS RAPIDEMENT SI BIEN QU'ON Ne PEUT PAS LE LIRE, IL APPARAIT PARFOIS PLUS LENTEMENT ET AVEC DES CARACTÈRES PLUS gRANDS ET L'ON EST PRESQUE OBLIgÉs À LE LIRE... QUELLE RÉFLEXION SUR L'ÉCRITURE MENAIT LE TRAVAIL ?

Patrick Zanoli : Dès le début, l'idée principale fut qu'il fallait que le texte soit présent tout le temps et qu'il défile, avec quelques arrêts pour des histoires de rythme. Un flux d'image et un flux de texte, c'était l'idée. On aurait pu imaginer que le film soit fait de plans fixes de texte ; or, là, il fallait que cela défile.

\section{C'ÉTAIT PLUS UNE QUESTION FORMELLE QU'UN RAPPORT À CE QUE RACONTAIT LE TEXTE ?}

Catherine Derosier-Pouchous : Les deux. En revoyant le film, je pense qu'il y a plusieurs narrations. D'abord celle de son rapport personnel aux gens à qui est dédié ce film qu'il n'a pas pu ou osé filmer - il m'a dit ne pas avoir osé leur demander, mais ce film est pour eux. Il y a quelque chose de très personnel, qui affleure de loin en loin quand il parle de Ron et de Gary. Il y a le récit de ce qu'on voit, les gens qu'il rencontre, sa vie, le travail de théâtre; puis le fil d'ironie et de distance autour de la maladie ; c'est très factuel mais, en même temps, c'est sous-tendu par des sensations, des sentiments de souffrance, de chagrin, de tristesse, de difficulté, d'angoisse, et cette espèce de pirouette en permanence autour de ces questions-là. Enfin, il y a tout le récit historique de ce qui se passe à Berlin, de ce qui se passe en France, des préoccupations qu'il a sur l'état du monde de l'époque, son fonctionnement politico-social. Alors, selon la manière dont on lit le texte, on suit plus ou moins un ou plusieurs de ces fils, et ce n'est pas grave si l'on manque un morceau du texte à l'image car comme il y a de la récurrence, ça revient. Le récit fonctionne avec ces quatre ou cinq fils qu'on suit : c'est donc à la fois une réflexion sur la forme, la forme de l'écrit à l'image, la circulation de l'écrit, mais aussi une façon de répéter, de reformuler, d'évoquer différentes choses.

François Berreur : Il y a aussi le fait qu'à partir du quotidien qu'il filme, il crée une sorte de mythologie, quelque chose qui, de la manière dont s'est monté et avec les images qu'il garde, ne correspond pas au réel. La manière dont il raconte le moment où l'on boit le café et que je sors de la douche, on a l'impression d'une espèce de communauté post baba, alors que c'est juste parce que je prends un avion tôt ! Au fond, on n'a jamais eu de vie communautaire, mais je le connais, il a plaisir à filmer cela de cette manière. Il y a aussi la scène de Mireille et moi qui répétons dans le canapé, il garde ces images-là et d'autres il ne les garde pas. On voit qu'il essaie de créer une petite mythologie de l'histoire qui ne correspond pas du tout à la manière dont on travaillait et répétait.

À Berlin, l'endroit où il habitait, j'y suis allé : en effet, c'était un appartement, du type ancienne caserne militaire, et c'est vrai que quand on voit la baie vitrée, là où on 
prenait le café, il n'y avait personne; c'était un ancien réfectoire. Il y avait des chambres en dortoir, avec deux ou trois lits. Or, de la manière dont il le filme, on dirait une sorte d'hôtel un peu mythique avec les avions qui décollent; c'est vrai que les avions décollaient, mais, quand on était à l'intérieur, c'était une sorte de vieux lycée abandonné terrifiant où il n'y avait rien. Le soir, il fallait prendre la voiture pour rentrer...

Les gens me demandent souvent comment il écrivait: dans le film il y a des images de lui qui écrit, et quand je regarde le film, je trouve que cela ne correspond à rien, c'est totalement aberrant. Les seules images un peu concrètes, c'est quand il y a la machine à écrire et c'était dans son appartement à Paris.

Catherine Derosier-Pouchous : On est au cinéma dans le sens où on est dans la fiction, avec une mise en scène, une narration qui est construite ; ce n'est pas un documentaire, pas un essai personnel, même si en même temps c'est très investi.

PARMI LES ÉlÉMENTS FRAPPANTS dU fILM, ON PEUT NOTER L'UTILISATION DE TOUT CE QUI DONNE DU MOUVEMENT: LA VOITURE, LE TRAIN, TOUS CES PANORAMIQUES, LE PLUS

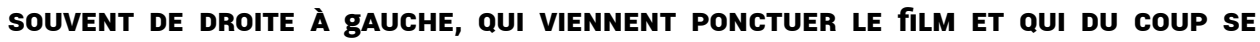
MÊLENT AVEC LE dÉfilement CONTINU dU TEXTE. AU MOMENT dU MONTAgE, C'ÉTAIT UNE VOLONTÉ IMPORTANTE CHEZ LUI ?

Patrick Zanoli : Il n'y avait aucune ambiguïté là-dessus : il avait choisi précisément tous les morceaux de rush qu'il voulait mettre dans son film. Nous avions des discussions sur la manière dont nous pouvions mélanger ces images, avec ce texte qui bouge aussi, car c'était complexe en termes de lecture, mais il avait précisément choisi les images qui sont dans le film. Pour certaines, il s'est un peu censuré, par exemple les enregistrements qu'il avait faits sur les quais à Austerlitz, et quelques autres images aussi.

À L'ÉPOQUe du CINÉmA MUET, dANS LeS ANNÉEs 1920, IL Y AVAIT EU UN CERTAIN NOMBRE DE filmS OPÉRANT DES MONTAgES EXPÉRIMENTAUX AVEC SUPERPOSITIONS D'IMAgES,

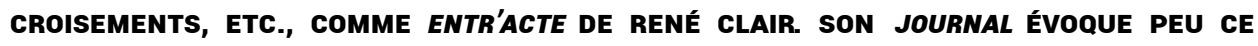
Cinéma. est-ce que cela faisait partie de ses références? la découverte des outils de montage lui A-t-elle permis de Retrouver ce sens du cinéma EXPÉRIMENTAL DES ANNÉES 1920 ?

François Berreur : D'une certaine manière, le Journal n'est pas du tout représentatif et n'a rien à voir avec Jean-Luc Lagarce, et notamment sa culture et son rapport avec elle. Il y écrit ce qu'il va voir, mais c'est comme sur l'écriture. Jean-Luc ne m'a jamais beaucoup parlé de Voltaire ou de la philosophie; pourtant il avait une solide culture philosophique. Il avait suivi à Besançon un cours de cinéma et il possédait une culture cinématographique aussi large que sa culture littéraire. Il avait vu énormément de films, et il connaissait très bien l'histoire du cinéma : c'est quelque chose qu'il avait acquis assez jeune, avec la fréquentation permanente de tous les cinémas de Besançon, des ciné-clubs, etc. C'est comme dans son écriture, où l'on voit aujourd'hui les références d'écrivain dans ses œuvres. Tout cela fait partie du bagage intellectuel.

Il disait que si l'on écrit quelque chose, c'est pour faire avec tout ce qu'on a de l'histoire de l'humanité, et en se demandant comment faire un pas de plus. Quand il fait le film, il a cette ambition-là : c'est d'une prétention extrême! Car l'idée, c'est la postérité; ce n'est pas une demi-ambition, c'est très présent quand il écrit. Est-ce que dans un siècle on lira encore Jean-Luc Lagarce? La douleur de l'échec de Juste la fin du monde correspond à ce point-là, cet endroit de reconnaissance. L'ambition de son travail sur le film est dans cette expérimentation, dans le rapport aux nouvelles technologies : c'était 
l'époque où l'on voyait les premières vidéos d'artistes en dehors de la télévision. Il mixe un rapport de recherche un peu pur, le rapport littéraire, et cette quête d'exploration de nouvelles pistes. La force du CICV, dans les années 1980, c'était d'être un lieu à la charnière de l'ancienne télévision, de l'ancienne image, et de la nouvelle vidéo prénumérique ; c'est une espèce d'endroit né presque trop tôt peut-être, avec beaucoup de liberté par rapport à des enjeux qui ont incroyablement évolué.

\section{NOUS AVONS DANS LES ARCHIVES DE LAgARCE DES DOSSIERS D'IMAgES ASSEZ IMPORTANTS

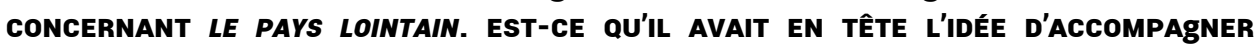 ÉVENTUELLEMENT LA REPRÉSENTATION DE PROJECTIONS ? EST-CE QUE CETTE IDÉE SERAIT ENTRÉE dANS SES HABITUdES DE METTEUR EN SCÈNE ?}

François Berreur : Cela, on ne peut pas trop savoir. Il avait sa documentation pour tous les spectacles; la documentation est liée non à l'écriture, mais au projet de représentation, puisque Le Pays Lointain, c'est une commande de François Le Pillouër pour le festival Mettre en scène. On a fait un spectacle en 1992 dans lequel il y avait des téléviseurs partout ; c'était Histoire d'amour, derniers chapitres. L'équipement était lourd, terrible, ça ne correspondait pas du tout au projet! Entre la réalisation du spectacle et le projet de Jean-Luc, les moyens étaient moyennement adéquats. Est-ce qu'il aurait interrogé d'autres formes par rapport à ça ? Oui, certainement. D'autant plus qu'Histoire d'amour était un endroit d'expérimentation puisqu'on devait le faire tous les dix ans !

\section{NOTES}

1. Centre International de Création Vidéo Pierre Schaeffer, basé à Hérimoncourt, dans le pays de Montbéliard, et qui a fermé en 2004.

\section{INDEX}

Mots-clés : Jean-Luc Lagarce, Juste la fin du monde, Le Pays lointain, François Berreur, Catherine Derosier-Pouchous, Patrick Zanoli

\section{AUTEURS}

\section{PASCAL LÉCROART}

Pascal Lécroart est professeur de Littérature française à l'Université de Franche-Comté où il dirige le Pôle « Arts et littérature » au sein d'ELLIADD (EA 4661). Spécialiste de Paul claudel et de son rapport à la musique, il a publié différentes études sur les relations entre littérature, théâtre 
et musique au $\mathrm{Xx}^{\mathrm{e}}$ siècle. Il est responsable du Fonds numérique d'archives Jean-Luc Lagarce hébergé par FANUM.

\section{ALEXIS LEPRINCE}

Alexis Leprince est doctorant boursier de la MSHE Claude Nicolas Ledoux à l'Université de Besançon Franche Comté où il poursuit une recherche en génétique sur l'œuvre théâtrale du dramaturge Jean-Luc Lagarce. Également praticien, il est le collaborateur régulier de la compagnie Artépo, en tant qu'assistant à la mise en scène et dramaturge. 